\title{
Grau de satisfação entre os usuários de uma unidade básica de saúde no estado do Paraná, Brasil
}

\author{
Degree of satisfaction among users of a basic health unit in the state of Paraná, Brazil
}

\author{
Kamilla Zampieri Rodrigues (D) 1, Camila Ferreira Pires Mattos (D) 1, Dariele Aparecida Ferreira (D) 1 \\ Luiza Foltran de Azevedo Koch (D) 1 , Ernesto Josué Schmitt (D) 1, Marilisa Carneiro Leão Gabardo (D) $1 \square$
}

${ }^{1}$ Universidade Positivo, Escola de Ciências da Saúde. Curitiba, Paraná.

Como citar este artigo (How to cite this article):

Rodrigues KZ, Mattos CFP, Ferreira DA, Koch LFA, Schmitt EJ, Gabardo MCL. Grau de satisfação entre os usuários de uma unidade básica de saúde no estado do Paraná, Brasil (Degree of satisfaction among users of a basic health unit in the state of Paraná, Brazil). Sci Med. 2018;28(4):ID32253. DOI: 10.15448/1980-6108.2018.4.32253

\section{RESUMO}

OBJETIVOS: Avaliar o grau de satisfação dos usuários com a estrutura e os serviços prestados em uma unidade básica do Sistema Único de Saúde.

MÉTODOS: Este estudo transversal incluiu indivíduos com idade $\geq 18$ anos, de ambos os gêneros, entrevistados individualmente de março a maio de 2018 na unidade básica de saúde Ferraria, em Campo Largo, Paraná, Brasil. Um questionário estruturado continha informações sociodemográficas e questões adaptadas do instrumento utilizado no Programa Nacional de Avaliação de Serviços de Saúde, acerca da satisfação com a equipe, tempo de espera e estrutura física local. A análise descritiva dos dados foi seguida dos testes U de Mann-Whitney, Kruskal-Wallis, Qui-quadrado e regressão de Poisson, considerando como significativo $p<0,05$.

RESULTADOS: A amostra foi constituída por 373 usuários que atenderam aos critérios de inclusão, sendo 72,9\% do gênero feminino, 82,8\% de raça/cor da pele branca, $69,0 \%$ com baixa renda e 36,4\% com baixa escolaridade. A equipe de saúde foi o item melhor avaliado (73,3\% das respostas nas categorias "muito bom" e "bom"); e o tempo de espera para o atendimento foi o item pior avaliado (61,5\% "ruim" ou "muito ruim"). $\mathrm{Na}$ análise bivariada o atendimento da equipe de saúde não foi associado a nenhuma variável independente. Na avaliação geral do estabelecimento, as categorias "muito bom/bom", "regular", "ruim" e "muito ruim" foram avaliadas respectivamente por 84 (27,4\%), 128 $(41,7 \%), 67(21,8 \%)$ e $28(9,1 \%)$ brancos; e $22(34,9 \%), 34(54,0 \%), 7(11,1 \%)$ e $0(0,0 \%)$ não brancos $(p=0,006)$. No modelo de regressão de Poisson, a baixa escolaridade foi associada a uma chance 1,5 vezes maior de não saber onde reclamar em caso de mau atendimento.

CONCLUSÕES: O grau de satisfação dos usuários da unidade básica de saúde Ferraria foi influenciado por fatores como raça/cor da pele e escolaridade. Os usuários não brancos expressaram um grau de satisfação maior do que os usuários brancos; e aqueles com baixa escolaridade sabiam menos onde reclamar caso o atendimento não fosse satisfatório. Apesar da avaliação em geral ter sido positiva, alguns quesitos foram identificados como não satisfatórios, merecendo medidas corretivas

DESCRITORES: estudos transversais; serviços de saúde; Sistema Único de Saúde; satisfação do usuário

\section{ABSTRACT}

AIMS: To evaluate the users' degree of satisfaction with the structure and services provided in a Basic Health Unit of the Public Health System. METHODS: This cross-sectional study included individuals aged $\geq 18$ years, of both genders, individually interviewed from March to May 2018 at the Ferraria Basic Health Unit, Campo Largo, Paraná, Brazil. A structured questionnaire contained sociodemographic information and questions adapted from the instrument used in the National Health Services Evaluation Program, regarding satisfaction with the staff, waiting time and local physical structure. Descriptive analysis of the data was followed by the Mann-Whitney U, Kruskal-Wallis, Chi-square and Poisson regression tests. Values of $\mathrm{p}<0.05$ were considered statistically significant.

RESULTS: The sample consisted of 373 users who met the inclusion criteria, of whom $72.9 \%$ were female, $82.8 \%$ were white race/skin color, $69.0 \%$ had low income and $36.4 \%$ had low schooling. The health team was the best evaluated item ( $73.3 \%$ of answers in the categories "very good" and "good"); and the waiting time for service was the worst evaluated item (61.5\% of answers "bad" or "very poor"). In the bivariate analysis, the health team care was not associated with any independent variables. In the general evaluation of the establishment, the categories "very good/good", "regular", "bad" and "very bad" were evaluated respectively by $84(27.4 \%), 128(41.7 \%), 67(21.8 \%)$ and 28 (9.1\%) white users; and $22(34.9 \%), 34(54.0 \%), 7(11.1 \%)$ and $0(0.0 \%)$ non-white users $(\mathrm{p}=0.006)$. In the Poisson regression model, low schooling was associated with a 1.5 times greater chance of not knowing where to complain in case of poor care.

CONCLUSIONS: The degree of satisfaction of the users of the Ferraria Basic Health Unit was influenced by factors such as race/skin color, income and schooling. Non-white users expressed a higher degree of satisfaction than white users. Users with low schooling knew less where to complain if service was not satisfactory. Although the evaluation was generally positive, some items were identified as unsatisfactory, deserving corrective measures.

KEYWORDS: cross-sectional studies; health services; Unified Health System; consumer behavior 
Abreviaturas: ESF, Estratégia Saúde da Família; OR, odds ratio; SUS, Sistema Único de Saúde; UBS, unidade básica de saúde.

\section{INTRODUÇÃO}

O Sistema Único de Saúde (SUS) tem como princípio a universalidade do acesso, a integralidade da assistência, a participação da comunidade, a descentralização político-administrativa, a regionalização, a hierarquização e a capacidade de resolução dos serviços em todos os níveis de atenção. Tratase de uma organização federativa fundamentada em uma rede de estabelecimentos públicos e em conjunto com estabelecimentos privados complementares, cuja participação colaborativa decorre dos governos federal, estadual e municipal [1].

O processo de descentralização do atendimento em saúde favorece a consolidação da municipalização da gestão do SUS, fazendo com que os municípios sejam os responsáveis pelo oferecimento da atenção básica a seus moradores, gerenciando os serviços de saúde de modo geral, seja no planejamento, na organização, no controle e na avaliação das ações de saúde, dentre outras incumbências [2]. Todas essas condições são necessárias para que sejam alcançadas resolutividade, qualidade e humanização por parte das ações e dos serviços prestados à população [3].

Cada vez mais os municípios assumem essa gestão descentralizada, passando a ser responsáveis pelo relacionamento com os prestadores de serviço. $\mathrm{O}$ Ministério da Saúde, por meio da Norma Operacional de Assistência à Saúde (NOAS SUS 01/2002) [4] institui duas condições de participação do município na gestão do SUS: Gestão Plena da Atenção Básica Ampliada - na qual o município recebe um valor definido em base per capita para o financiamento da atenção básica; e Gestão Plena do Sistema Municipal - na qual o município recebe o total de recursos provenientes do governo federal programados para o custeio da assistência em seu território. Dentre as prerrogativas da primeira fica estabelecida e "gestão de todas as unidades básicas de saúde, públicas ou privadas (lucrativas e filantrópicas) integrantes do SUS, localizadas no território municipal". Já na segunda, a "gerência de unidades próprias, ambulatoriais e hospitalares" também é citada como responsabilidade $[4,5]$.

De modo a reorganizar a prática de atenção à saúde e assim consolidar os princípios do SUS e a expansão da atenção primária em saúde [6], o Ministério da Saúde propôs aos municípios a criação do Programa de Saúde da Família, atualmente denominado Estratégia Saúde da Família (ESF) [7, 8]. A ESF está pautada, dentre outras diretrizes, no trabalho em equipe multiprofissional e na participação social $[7,8]$. Nesse sentido, a voz da comunidade se expressa nas decisões tomadas pelo Estado, sendo o cidadão/usuário o centro desse processo de avaliação $[9,10]$. Esse controle pressupõe o usuário como potencial interventor e modificador do sistema [11], o que se reflete como fortalecimento da democracia em saúde [12]. Ainda, a humanização do serviço também é alcançada, bem como a oportunidade de se verificar, na prática, a resposta da comunidade à oferta do serviço de saúde, e como esse processo se dá de modo inverso [13]. Experiências exitosas em termos de aumento da satisfação dos usuários quando da implementação da ESF foram constatadas em diversos municípios do país [10,14-19], em contraposição a achados menos favoráveis que apontam para a necessidade de melhorias em vários processos [20,21].

As deficiências na relação população-profissional são reconhecidas, e pesquisadores afirmam que a participação crítica dos sujeitos contribui de algum modo e em algum nível para a viabilização de políticas de saúde [17,20,22,23]. Neste caso, somando-se as questões de ambiente e processos de trabalho, faz-se necessária a avaliação da satisfação dos usuários do SUS, como um importante balizador para o planejamento e melhorias no sistema de saúde [10,20,24]. Essa satisfação pode ser determinada, basicamente, pelos seguintes fatores: características dos usuários e dos profissionais, relação estabelecida entre os mesmos, e fatores relacionados ao local, como estrutura e ambiente $[15,25]$.

Estudos têm sido conduzidos no sentido de avaliar a percepção dos usuários a respeito dos serviços de saúde, em nível nacional [10,16, 19,26-28] e internacional [29-32]. Essas pesquisas apontam com principais aspectos negativos a falta de tempo despendido por parte dos profissionais [27,29] e problemas com infraestrutura [27].

De acordo com o exposto, esse artigo teve como objetivos caracterizar o perfil sociodemográfico e avaliar o grau de satisfação dos usuários com os serviços prestados na unidade básica de saúde (UBS) Ferraria, na cidade de Campo Largo, Paraná, Brasil.

\section{MÉTODOS}

Esta pesquisa foi aprovada pelo Comitê de Ética em Pesquisa com Seres Humanos da Universidade Positivo, sob registro no 2.227 .943 . 
Campo Largo está localizada na Região Metropolitana de Curitiba e conta aproximadamente 125.719 habitantes [33]. A elevada densidade demográfica (aproximadamente 15.739 habitantes), somada à expansão urbana desorganizada e irregular, posiciona o Distrito Ferraria como uma área de cuidado prioritário [34]. Na região há uma UBS com ESF, a qual conta com cinco médicos, duas enfermeiras, três técnicos de enfermagem, uma auxiliar de enfermagem, dois cirurgiões-dentistas, duas auxiliares em saúde bucal, uma auxiliar de farmácia e nove agentes comunitários de saúde. Há também profissionais do Núcleo de Apoio à Saúde da Família, com: dois fisioterapeutas, uma psicóloga, uma terapeuta ocupacional e uma assistente social. No local há placas com a sinalização da Ouvidoria do Município, informando as formas de contato para os usuários poderem reclamar caso não estejam satisfeitos com algum atendimento.

Para o cálculo amostral considerou-se a população de aproximadamente 12.000 usuários cadastrados na UBS, conforme informações da autoridade sanitária local. Sendo estimado o erro de $5 \%$ e nível de confiança de $95 \%$, a amostra foi calculada em 373 usuários. Com desenho transversal, foram incluídos usuários de ambos os gêneros, que preencheram os critérios de inclusão: idade igual ou superior a 18 anos, residentes no município, sem plano de saúde complementar e que assinaram o Termo de Consentimento Livre e Esclarecido.

Para a seleção da amostra foram sorteados os prontuários daqueles que haviam acabado de ser atendidos na UBS e se encontravam no local para a realização de exames, novas consultas, aquisição de medicamentos na farmácia ou que estavam fazendo novos agendamentos de atendimento. Atendidos os critérios de inclusão, o participante era conduzido a uma sala reservada na UBS. Esse processo foi feito de março a maio de 2018 até que se atingisse a amostra estimada. Durante toda a coleta, 84 pacientes se negaram a participar.

Optou-se pela realização de entrevistas individuais, com perguntas estruturadas, realizadas por três pesquisadores treinados previamente. Foram adaptadas questões do instrumento de 2015 do Programa Nacional de Avaliação de Serviços de Saúde, que visa avaliar os estabelecimentos de saúde, ambulatoriais ou hospitalares, que tenham financiamento do Ministério da Saúde [35].

A primeira parte do questionário foi composta de informações sobre o perfil sociodemográfico do entrevistado, a saber: idade (em anos), gênero, raça/ cor (autorreferida), estado civil, escolaridade e renda familiar. Para as análises multivariadas a idade, a renda e escolaridade foram categorizadas, sendo que raça/cor da pele e estado civil foram dicotomizadas. A segunda parte consistiu na avaliação do usuário em relação ao atendimento na UBS. Todas as questões do instrumento original referentes a roupas, alimentação e tratamento dado ao acompanhante foram removidas, visto que aquele inclui pacientes internados, o que não se aplica ao presente estudo.

Como desfechos principais foram eleitas as questões que mais simbolizaram o objetivo da pesquisa: 1) "Como o(a) senhor(a) avalia o atendimento da equipe de saúde?"; 2) "De uma maneira geral, como o(a) senhor(a) avalia este estabelecimento de saúde?" e 3 ) " $O(A)$ senhor(a) sabe onde reclamar quando não é bem atendido?". Para fins de análise, as respostas da primeira questão foram categorizadas em muito bom; bom; regular; ruim/muito ruim; para a segunda questão, as respostas foram divididas em muito bom/ bom; regular; ruim; muito ruim. A terceira contemplava apenas duas respostas possíveis: sim ou não.

Para as análises bivariadas foram utilizados os testes U de Mann-Whitney, Kruskal-Wallis ou Quiquadrado. Variáveis com valor de $\mathrm{p}<0,20$ nesses testes foram incluídas na regressão de Poisson. Todas as análises foram realizadas por meio do programa SPSS IBM versão 21.0 (Chicago, IL, EUA). Valores de $p<0,05$ foram considerados estatisticamente significativos.

\section{RESULTADOS}

Um total de 373 indivíduos participaram da pesquisa, com média de idade de 46,1 $\pm 16,6$ anos (mínima de 18 e máxima de 89 anos). Na Tabela 1 está descrita a distribuição das variáveis sociodemográficas. A amostra final foi composta por $72,9 \%$ de mulheres e $27,1 \%$ de homens. Os que se consideraram brancos foram $82,8 \%$, e houve predomínio da renda familiar entre um e dois salários mínimos $(69,0 \%)$.

Na Tabela 2 encontra-se a descrição das respostas às perguntas feitas por meio do questionário. Verificou-se que a maioria $(82,9 \%)$ dos usuários sentiu confiança e segurança na equipe de saúde durante o atendimento.

$\mathrm{Na}$ análise bivariada (Tabela 3), quando o desfecho era a pergunta (1), "Como o(a) senhor(a) avalia o atendimento da equipe de saúde?", não houve diferença em relação a nenhuma variável independente. Para a pergunta (2), "De uma maneira geral, como o(a) senhor(a) avalia este estabelecimento de 
saúde?", apenas raça/cor da pele apresentou diferença significativa $(\mathrm{p}=0,006)$, com os usuários não brancos tendendo a avaliar melhor do que os brancos. Quando analisado o desfecho (3), "O(A) senhor(a) sabe onde reclamar quando não é bem atendido?", as pessoas sabiam menos onde reclamar quanto mais baixa a renda $(\mathrm{p}=0,021)$ e mais baixa a escolaridade $(\mathrm{p}=0,004)$.

Quando incluídas essas duas variáveis no modelo de regressão de Poisson, apenas a baixa escolaridade manteve-se associada ao não saber onde reclamar, aumentando em 1,59 vezes a chance dessa ocorrência (Tabela 4).

Tabela 1. Frequências relativa e absoluta das características sociodemográficas dos usuários da unidade básica de saúde Ferraria, Campo Largo/PR, Brasil, participantes do estudo $(n=373)$.

\begin{tabular}{|c|c|c|}
\hline Característica & $\mathbf{n}$ & $\%$ \\
\hline \multicolumn{3}{|l|}{ Gênero } \\
\hline Masculino & 101 & 27,1 \\
\hline Feminino & 272 & 72,9 \\
\hline \multicolumn{3}{|l|}{ Raça/Cor da pele } \\
\hline Branca & 309 & 82,8 \\
\hline Amarela & 3 & 0,8 \\
\hline Parda & 46 & 12,3 \\
\hline Negra/Preta & 15 & 4,0 \\
\hline \multicolumn{3}{|l|}{ Estado civil } \\
\hline Casado(a) & 202 & 54,6 \\
\hline União estável & 21 & 5,7 \\
\hline Viúvo(a) & 40 & 10,8 \\
\hline Divorciado(a) & 12 & 3,2 \\
\hline Separado(a) & 5 & 1,4 \\
\hline Solteiro(a) & 90 & 24,3 \\
\hline \multicolumn{3}{|l|}{ Escolaridade } \\
\hline Especialização/residência & 1 & 0,3 \\
\hline Superior completo & 6 & 1,6 \\
\hline Superior incompleto & 7 & 1,9 \\
\hline Médio completo & 104 & 28,0 \\
\hline Médio completo - magistério & 1 & 0,3 \\
\hline Médio completo - magistério indígena & 2 & 0,5 \\
\hline Médio incompleto & 63 & 17,0 \\
\hline Fundamental completo & 40 & 10,8 \\
\hline Fundamental incompleto & 135 & 36,4 \\
\hline Apenas alfabetizado & 5 & 1,3 \\
\hline Não sabe ler/escrever & 7 & 1,9 \\
\hline \multicolumn{3}{|l|}{ Renda familiar } \\
\hline Mais de 10 salários mínimos & 2 & 0,5 \\
\hline Mais de 5 a até 10 salários mínimos & 1 & 0,3 \\
\hline Mais de 2 e até 5 salários mínimos & 80 & 21,7 \\
\hline Entre 1 e 2 salários mínimos & 254 & 69,0 \\
\hline Menos de 1 salário mínimo & 13 & 3,5 \\
\hline Não tem renda & 18 & 4,9 \\
\hline
\end{tabular}

Tabela 2. Frequências relativa e absoluta das perguntas feitas aos usuários da unidade básica de saúde Ferraria, Campo Largo/PR, Brasil, participantes do estudo $(n=373)$.

\begin{tabular}{|c|c|c|}
\hline Pergunta & $\mathbf{n}$ & $\%$ \\
\hline \multicolumn{3}{|c|}{$\begin{array}{l}\text { Para ser atendido neste estabelecimento de saúde, } \\
\text { o(a) senhor(a): }\end{array}$} \\
\hline Precisou agendar/marcar o procedimento & 99 & 26,8 \\
\hline Foi atendimento de emergência & 270 & 73,2 \\
\hline \multicolumn{3}{|c|}{$\begin{array}{l}\text { Quanto tempo o(a) senhor(a) esperou para marcar este } \\
\text { procedimento? }\end{array}$} \\
\hline Até 15 dias & 73 & 73,8 \\
\hline De 16 a 30 dias & 13 & 13,1 \\
\hline De 31 a 60 dias & 11 & 11,1 \\
\hline De 61 a 90 dias & 1 & 1,0 \\
\hline De 6 meses a 1 ano & 1 & 1,0 \\
\hline \multicolumn{3}{|c|}{$\begin{array}{l}\text { O(A) senhor(a) considera que esse tempo de espera } \\
\text { para marcar o procedimento foi: }\end{array}$} \\
\hline Muito bom & 5 & 4,7 \\
\hline Bom & 32 & 30,2 \\
\hline Regular & 35 & 33,0 \\
\hline Ruim & 26 & 24,6 \\
\hline Muito ruim & 8 & 7,5 \\
\hline \multicolumn{3}{|c|}{$\begin{array}{l}\text { No dia do procedimento, quanto tempo o(a) senhor(a) } \\
\text { levou para ser atendido dentro do serviço? }\end{array}$} \\
\hline Até 30 minutos & 28 & 7,6 \\
\hline Até 1 hora & 55 & 14,9 \\
\hline Até 2 horas & 60 & 16,3 \\
\hline Até 4 horas & 161 & 43,6 \\
\hline Até 12 horas & 65 & 17,6 \\
\hline \multicolumn{3}{|c|}{$\begin{array}{l}\text { O(A) senhor(a) considera que esse tempo de espera } \\
\text { para ser atendido foi: }\end{array}$} \\
\hline Muito bom & 3 & 0,8 \\
\hline Bom & 49 & 13,2 \\
\hline Regular & 91 & 24,5 \\
\hline Ruim & 108 & 29,0 \\
\hline Muito ruim & 121 & 32,5 \\
\hline \multicolumn{3}{|l|}{$\begin{array}{l}\text { No geral, como o(a) senhor(a) julga a limpeza dos } \\
\text { ambientes? }\end{array}$} \\
\hline Muito bom & 9 & 2,4 \\
\hline Bom & 174 & 46,6 \\
\hline Regular & 141 & 37,8 \\
\hline Ruim & 32 & 8,6 \\
\hline Muito ruim & 17 & 4,6 \\
\hline \multicolumn{3}{|c|}{$\begin{array}{l}\text { Como o(a) senhor(a) avalia a sinalização do estabele- } \\
\text { cimento para encontrar o local onde precisa ir? }\end{array}$} \\
\hline Muito bom & 8 & 2,2 \\
\hline Bom & 233 & 63,7 \\
\hline Regular & 82 & 22,4 \\
\hline Ruim & 32 & 8,7 \\
\hline Muito ruim & 11 & 3,0 \\
\hline \multicolumn{3}{|c|}{ Como o(a) senhor(a) avalia o atendimento da recepção? } \\
\hline Muito bom & 32 & 8,6 \\
\hline Bom & 226 & 60,9 \\
\hline Regular & 90 & 24,3 \\
\hline Ruim & 14 & 3,8 \\
\hline Muito ruim & 9 & 2,4 \\
\hline
\end{tabular}


Tabela 2 (continuação)

\begin{tabular}{|c|c|c|c|c|c|}
\hline Pergunta & $\mathbf{n}$ & $\%$ & Pergunta & $\mathbf{n}$ & $\%$ \\
\hline $\begin{array}{l}\text { Como o(a) senhor(a) avalia o atendimento da equipe } \\
\text { de saúde? }\end{array}$ & & & $\begin{array}{l}\text { De uma maneira geral, como o(a) senhor(a) avalia este } \\
\text { estabelecimento de saúde? }\end{array}$ & & \\
\hline Muito bom & 45 & 12,2 & Muito bom & 5 & 1,4 \\
\hline Bom & 226 & 61,1 & Bom & 101 & 27,3 \\
\hline Regular & 83 & 22,3 & Regular & 164 & 44,3 \\
\hline Ruim & 11 & 3,0 & Ruim & 72 & 19,4 \\
\hline Muito ruim & 5 & 1,4 & Muito ruim & 28 & 7,6 \\
\hline $\begin{array}{l}\text { O(A) senhor(a) sentiu segurança/confiança na equipe } \\
\text { de saúde durante o atendimento? }\end{array}$ & & & $\begin{array}{l}\text { O(A) senhor(a) sabe onde reclamar quando não é } \\
\text { bem atendido? }\end{array}$ & & \\
\hline Sim & 287 & 82,9 & Sim & 144 & 38,6 \\
\hline Não & 59 & 17,1 & Não & 229 & 61,4 \\
\hline \multirow{2}{*}{\multicolumn{3}{|c|}{$\begin{array}{l}\text { Como o(a) senhor(a) avalia as informações e } \\
\text { esclarecimentos que você teve sobre o seu estado de } \\
\text { saúde no estabelecimento? }\end{array}$}} & $\begin{array}{l}\mathrm{O}(\mathrm{A}) \text { senhor(a) pagou por algum valor durante este } \\
\text { procedimento? }\end{array}$ & & \\
\hline & & & Não & 373 & 100,0 \\
\hline Muito bom & 29 & 7,9 & $\operatorname{Sim}$ & 0 & 0,0 \\
\hline Bom & 227 & 62,0 & \multirow{2}{*}{\multicolumn{3}{|c|}{$\begin{array}{l}\text { O(A) senhor(a) sabe o nome do profissional de saúde } \\
\text { que lhe atendeu? }\end{array}$}} \\
\hline Regular & 82 & 22,4 & & & \\
\hline Ruim & 20 & 5,5 & Sim & 289 & 78,3 \\
\hline Muito ruim & 8 & 2,2 & Não & 80 & 21,7 \\
\hline
\end{tabular}

Tabela 3. Resultados da análise bivariada para os desfechos relatados por usuários da UBS Ferraria Campo Largo/PR, Brasil $(n=373)$, na avaliação do atendimento da equipe de saúde, do estabelecimento de saúde e do saber onde reclamar mediante mau atendimento.

\begin{tabular}{|c|c|c|c|c|c|c|c|c|c|c|c|c|c|}
\hline \multirow{3}{*}{ Característica } & \multicolumn{5}{|c|}{$\begin{array}{l}\text { 1) Como o(a) senhor(a) avalia o atendimento da } \\
\text { equipe de saúde? }\end{array}$} & \multicolumn{5}{|c|}{$\begin{array}{l}\text { 2) De uma maneira geral, como o(a) senhor(a) avalia } \\
\text { este estabelecimento de saúde? }\end{array}$} & \multicolumn{3}{|c|}{$\begin{array}{l}\text { 3) } \mathrm{O}(\mathrm{A}) \text { senhor(a) sabe onde } \\
\text { reclamar quando não é bem } \\
\text { atendido? (sim ou não) }\end{array}$} \\
\hline & $\begin{array}{c}\text { Muito } \\
\text { bom }\end{array}$ & Bom & Regular & $\begin{array}{l}\text { Ruim/ } \\
\text { muito } \\
\text { ruim }\end{array}$ & $\mathrm{p}$ & $\begin{array}{l}\text { Muito } \\
\text { bom/ } \\
\text { bom }\end{array}$ & Regular & Ruim & $\begin{array}{l}\text { Muito } \\
\text { ruim }\end{array}$ & $\mathrm{p}$ & Sim & Não & $\mathrm{p}$ \\
\hline & n (\%) & n (\%) & n (\%) & n (\%) & & n (\%) & n (\%) & n (\%) & n (\%) & & n (\%) & n (\%) & \\
\hline \multicolumn{14}{|l|}{ Idade } \\
\hline $18-32$ & $14(15,1)$ & $49(52,7)$ & $23(24,7)$ & $7(7,5)$ & $0,473^{*}$ & $28(29,8)$ & $32(34,0)$ & $22(23,4)$ & $12(12,8)$ & $0,051^{*}$ & $43(45,7)$ & $51(54,3)$ & $0,147^{\dagger}$ \\
\hline $33-48$ & $11(11,2)$ & $65(66,3)$ & $19(19,4)$ & $3(3,1)$ & & $22(22,2)$ & $52(52,5)$ & $18(18,2)$ & $7(7,1)$ & & $41(41,0)$ & $59(59,0)$ & \\
\hline $49-60$ & $10(11,4)$ & $52(59,1)$ & $21(23,9)$ & $5(5,7)$ & & $27(30,7)$ & $34(38,6)$ & $23(26,1)$ & $4(4,5)$ & & $33(37,5)$ & $55(62,5)$ & \\
\hline $61-89$ & $10(11,0)$ & $60(65,9)$ & $20(22,0)$ & $1(1,1)$ & & $29(32,6)$ & $44(49,4)$ & $11(12,4)$ & $5(5,6)$ & & $27(29,7)$ & $64(70,3)$ & \\
\hline \multicolumn{14}{|l|}{ Gênero } \\
\hline Masculino & $11(11,0)$ & $54(54,0)$ & $32(32,0)$ & $3(3,0)$ & $0,086^{\ddagger}$ & $31(31)$, & $47(47,0)$ & $17(17,0)$ & $5(5,0)$ & $0,199 \ddagger$ & $36(35,6)$ & $65(64,4)$ & $0,276^{*}$ \\
\hline Feminino & $34(12,6)$ & $172(63,7)$ & $51(18,9)$ & $13(4,8)$ & & $75(27,8)$ & $115(42,6)$ & $57(21,1)$ & $23(8,5)$ & & $108(39,7)$ & $164(60,3)$ & \\
\hline \multicolumn{14}{|l|}{ Raça/Cor da pele } \\
\hline Branco & $39(12,7)$ & $179(59,3)$ & $74(24,1)$ & $15(4,9)$ & $0,172^{\ddagger}$ & $84(27,4)$ & $128(41,7)$ & $67(21,8)$ & $28(9,1)$ & $0,006^{\ddagger}$ & $120(38,8)$ & $189(61,2)$ & $0,479 *$ \\
\hline Não branco & $6(9,5)$ & $47(74,6)$ & $9(14,3)$ & $1(1,6)$ & & $22(34,9)$ & $34(54,0)$ & $7(11,1)$ & $0(0,0)$ & & $24(37,5)$ & $40(62,5)$ & \\
\hline \multicolumn{14}{|l|}{ Estado civil } \\
\hline Casado & $24(10,9)$ & $132(60,0)$ & $54(24,5)$ & $10(4,5)$ & $0,225^{\ddagger}$ & $56(25,3)$ & $100(45,2)$ & $51(23,1)$ & $14(6,3)$ & $0,139 \neq$ & $92(41,3)$ & $131(58,7)$ & $0,123^{*}$ \\
\hline Não casado & $20(13,6)$ & $92(62,6)$ & $29(19,7)$ & $6(4,1)$ & & $50(34,2)$ & $60(41,1)$ & $22(15,1)$ & $14(9,6)$ & & $51(34,7)$ & $96(65,3)$ & \\
\hline \multicolumn{14}{|l|}{ Renda familiar } \\
\hline >2 salários mínimos & $13(15,7)$ & $51(61,4)$ & $16(19,3)$ & $3(3,6)$ & $0,264 t$ & $30(36,1)$ & $33(39,8)$ & $13(15,7)$ & $7(8,4)$ & $0,203+$ & $42(50,6)$ & $41(49,4)$ & $0,021+$ \\
\hline 1 a 2 salários mínimos & $27(10,8)$ & $152(60,6)$ & $59(23,5)$ & $13(5,2)$ & & $65(25,9)$ & $116(46,2)$ & $54(21,5)$ & $16(6,4)$ & & $92(36,2)$ & $162(63,8)$ & \\
\hline <1 salário mínimo & $5(16,1)$ & $19(61,3)$ & $7(22,6)$ & $0(0,0)$ & & $8(25,8)$ & $11(35,5)$ & $7(22,6)$ & $5(16,1)$ & & $8(25,8)$ & $23(74,2)$ & \\
\hline \multicolumn{14}{|l|}{ Escolaridade } \\
\hline EM completo a PG & $15(12,4)$ & $75(62,0)$ & $28(23,1)$ & $3(2,5)$ & $0,754 \boldsymbol{t}$ & $35(28,9)$ & $52(43,0)$ & $24(19,8)$ & $10(8,3)$ & $0,939 \dagger$ & $62(51,2)$ & $59(48,8)$ & $0,004^{\dagger}$ \\
\hline $\begin{array}{l}\text { EM incompleto } \\
\text { a EF completo }\end{array}$ & $16(15,7)$ & $59(57,8)$ & $23(22,5)$ & $4(3,9)$ & & $29(28,4)$ & $42(41,2)$ & $25(24,5)$ & $6(5,9)$ & & $34(33,0)$ & $69(67,0)$ & \\
\hline $\begin{array}{l}\text { Alfabetizado } \\
\text { a EF incompleto }\end{array}$ & $12(8,7)$ & $88(63,8)$ & $30(21,7)$ & $8(5,8)$ & & $38(27,5)$ & $65(47,1)$ & $23(16,7)$ & $12(8,7)$ & & $46(32,9)$ & $94(67,1)$ & \\
\hline Não alfabetizado & $1(14,3)$ & $4(57,1)$ & $1(14,3)$ & $1(14,3)$ & & $3(42,9)$ & $2(28,6)$ & $2(28,6)$ & $0(0,0)$ & & $1(14,3)$ & $6(85,7)$ & \\
\hline
\end{tabular}

SM, salário(s) mínimo(s); EM, ensino médio; PG, pós-graduação; EF, ensino fundamental

* Qui-quadrado; † Teste de Kruskal-Wallis; ₹ Teste U de Mann-Whitney. 
Tabela 4. Resultados do modelo de regressão de Poisson para o desfecho "O (a) senhor (a) sabe onde reclamar quando não é bem atendido?" por parte dos usuários da unidade básica de saúde Ferraria, Campo Largo/PR, Brasil, participantes do estudo $(\mathrm{n}=373)$.

\begin{tabular}{|c|c|c|c|c|}
\hline \multirow[b]{2}{*}{ Característica } & \multicolumn{4}{|c|}{ O(A) senhor(a) sabe onde reclamar quando não é bem atendido? } \\
\hline & $\underset{\text { nim }}{\text { Sim) }}$ & $\begin{array}{l}\text { Não } \\
\text { n (\%) }\end{array}$ & $\mathbf{p}$ & $\begin{array}{c}\text { RP } \\
\text { (IC 95\%) }\end{array}$ \\
\hline \multicolumn{5}{|l|}{ Renda familiar } \\
\hline > 2 salários mínimos & $42(50,6)$ & $41(49,4)$ & - & 1 \\
\hline 1 a 2 salários mínimos & $92(36,2)$ & $162(63,8)$ & 0,155 & $1,18(0,94-1,51)$ \\
\hline$<1$ salário mínimo & $8(25,8)$ & $23(74,2)$ & 0,057 & $1,34(0,99-1,80)$ \\
\hline \multicolumn{5}{|l|}{ Escolaridade } \\
\hline Ensino médio completo a pós-graduação & $62(51,2)$ & $59(48,8)$ & - & 1 \\
\hline Ensino médio incompleto a fundamental completo & $34(33,0)$ & $69(67,0)$ & 0,012 & $1,35(1,07-1,70)$ \\
\hline Alfabetizado a fundamental incompleto & $46(32,9)$ & $94(67,1)$ & 0,015 & $1,32(1,05-1,65)$ \\
\hline Não alfabetizado & $1(14,3)$ & $6(85,7)$ & 0,024 & $1,59(1,06-2,39)$ \\
\hline
\end{tabular}

RP, razão de prevalência; IC, intervalo de confiança.

\section{DISCUSSÃO}

Este estudo identificou que a maioria dos usuários da UBS Ferraria considerou a estrutura e o serviço nas categorias entre bom e regular. Os aspectos positivos mais relevantes foram quanto ao relacionamento com a equipe de saúde, que tiveram a maioria das respostas em bom ou muito bom. Aspectos negativos foram mais relacionados a questões estruturais e de processo de trabalho. Essa percepção reduzida em termos de satisfação vai ao encontro de um cenário experienciado comumente no Brasil, onde a população não considera que os serviços de saúde atendam satisfatoriamente às suas demandas $[10,19,26]$.

AESF, dentre outros objetivos, destina-se a melhorar a satisfação do usuário, dada a maior integração serviçocomunidade, o que fortalece o controle social, princípio legalmente instituído pelo SUS $[9,10]$. Esse tipo de análise que inclui o usuário no centro do processo avaliativo, como agente transformador, é fundamental para permitir um redirecionamento de estratégias de gestão da saúde pública [9-13,29]. No Brasil e no mundo, investigações com diferentes métodos têm sido feitas com esse propósito [10,12, 19, 26-32].

No Brasil, a iniciativa federal denominada Programa Nacional de Melhoria do Acesso e da Qualidade da Atenção Básica (PMAQ-AB), que tem como proposta melhorar os serviços públicos de saúde, bem como a satisfação dos usuários, identificou que nas macrorregiões os fatores que influenciaram a satisfação foram: qualidade de acesso, atendimentos às necessidades e organização do processo de trabalho [19]. Os mesmos autores indicaram que na região sul a maior insatisfação relatada deveu-se ao descompromisso percebido pelos usuários [19]. A necessidade de maior atenção por parte do profissional durante as consultas foi apontada em outra pesquisa com usuários de uma UBS com ESF em um município de grande porte na região sudeste [36].

A satisfação dos usuários está relacionada a uma série de fatores, dentre os quais a relação profissionalpaciente e a estrutura física local [12,24]. A melhor percepção relacionada ao vínculo e à humanização pode ser ressaltada pelos pacientes, o que pode levá-los a minimizar questões referentes à capacidade de resolução dos problemas de saúde $[12,15]$. Esse aspecto não ocorreu completamente na amostra aqui estudada; quando questionados sobre o atendimento da esquipe de saúde, mais de $70 \%$ o classificaram como muito bom/bom, e a confiança na equipe de saúde foi manifestada por mais de $80 \%$. Entretanto, quando questionados sobre a qualidade em geral do estabelecimento, menos de $30 \%$ responderam nas categorias muito bom ou bom. Além disso, não foram encontradas diferenças significativas em relação às variáveis independentes (idade, gênero, raça/cor da pele, estado civil, renda e escolaridade) no que se refere à avaliação da equipe; entretanto, houve diferenças nas respostas que se referiam à avaliação geral do estabelecimento de saúde. A percepção positiva de usuários da ESF em relação à equipe de saúde foi relatada também, em diferentes épocas, por Sena et al. [16] em Recife, Pernambuco, Moimaz et al. [10] em cinco municípios do estado de São Paulo, Cotta et al. [12] em Teixeiras, Minas Gerais, e Jorge et al. [15] em uma amostra representativa de municípios do estado do Ceará.

Existe uma tendência de os usuários em condições menos favorecidas socioeconomicamente avaliarem melhor os serviços de saúde [37], condição denominada 
de "fenômeno da elevação na taxa de satisfação" [26]. Esse fato, entretanto, não foi encontrado na presente pesquisa, visto que mesmo em um contexto desfavorável sob o ponto de vista econômico, a insatisfação foi reportada com mais frequência.

A média de idade (46,1 anos) dos usuários, encontrada nesta pesquisa, aproxima-se dos valores relatados por outros autores, entre 40 e 60 anos $[12,18]$. A maior prevalência de mulheres também já foi relatada $[12,15,18,38]$. Segundo Bicalho et al. [39], a mulher é, na família, quem centraliza as questões de saúde e doença. É ela quem avalia sua própria condição de saúde e as dos demais membros da família, e procura os profissionais aptos a resolver cada caso [40]. Outra vertente atribui o cuidar como uma tarefa feminina [41]. Na literatura há evidências de que as mulheres são mais preocupadas com o autocuidado e, assim, procuram mais os serviços de saúde [42], da mesma forma que são mais capazes de fazer críticas [43].

$\mathrm{Na}$ amostra pesquisada, os brancos compuseram a maioria e foram os que mais se queixaram do estabelecimento de saúde como um todo e de modo significativo. Deve-se esclarecer que Campo Largo é um município de colonização europeia, o que determina a maior parte da população ser composta por indivíduos brancos [34]. Entretanto, não se pode deixar de mencionar que em populações brasileiras é comum que os indivíduos não brancos sejam os que menos têm acesso aos serviços de saúde [44]. Os casados, que também foram maioria, assim como em outras abordagens [38], podem ter tido esse comportamento pelo fato de o cônjuge, em especial as mulheres, induzirem e até mesmo acompanharem o esposo (ou a esposa) para o atendimento.

Os dados aqui encontrados indicaram maior prevalência de entrevistados com baixa escolaridade e baixa renda familiar, corroborando com outros achados $[12,18,38]$. Vale ressaltar que os participantes eram usuários exclusivos do SUS, sem qualquer tipo de atendimento em saúde suplementar, conforme os critérios de inclusão adotados. Melhores condições de renda familiar mensal e de escolaridade e maior frequência a consultas foram associados ao maior grau de satisfação de usuários da ESF em um município de Minas Gerais [12]. Santiago et al. [38], em pesquisa com 939 usuários de uma UBS com ESF no Recife, indicaram que os mais jovens e com escolaridade mais alta tiveram maior insatisfação com as condições oferecidas pelo serviço.

Com referência à questão " $\mathrm{O}(\mathrm{A})$ senhor(a) sabe onde reclamar quando não é bem atendido?", indivíduos com baixa escolaridade tiveram chance uma vez e meia maior de não saberem a quem reclamar. Os pesquisadores localizaram na UBS Ferraria placas com a sinalização da Ouvidoria do Município, o que ressalta a necessidade de melhoria da comunicação verbal com as pessoas não acostumadas a ler avisos com atenção (ou mesmo não capazes de ler), para que tomem ciência dos seus direitos enquanto usuários do SUS.

Constatou-se que mais de $70 \%$ dos pesquisados foram atendidos em caráter emergencial e o tempo para tanto foi de até quatro horas, julgado pelos mesmos como muito ruim. Esses dados confirmam a hipótese de que as pessoas tendem a procurar o serviço quando estão doentes e não costumam aderir a estratégias preventivas longitudinais, pressuposto recomendado na atenção primária em saúde, a qual teria capacidade de resolver até $80 \%$ dos problemas de saúde [6].

Outra informação interessante foi que os entrevistados relataram que alguns procedimentos agendados suprem as necessidades dos mesmos como, por exemplo, exame de sangue, urina e fezes, mas que esse tempo poderia ser reduzido. Contudo, a demora para o agendamento (15 dias) não foi classificada com um dos maiores problemas pelos usuários na presente pesquisa, situação que costuma ser bastante negativa em outros locais [36].

No estudo de Arruda e Bosi [17], 43 usuários de uma UBS foram entrevistados em suas próprias residências e afirmaram que o diálogo nas práticas assistenciais da saúde pública é de extrema importância, e que quando ausente, aumenta a insatisfação dos usuários. Observou-se, na presente pesquisa, que 78,3\% dos usuários relataram saber o nome de quem os atendeu. Esse vínculo expressivo também foi identificado por Campos et al. [36] e Santiago et al. [38]. Segundo Viegas et al. [45], embora a dimensão "operacional" seja determinante para o acesso a um serviço, a dimensão "relacional" tem papel fundamental na sua avaliação.

Ao se tratar de nível de satisfação é importante considerar os aspectos subjetivos da análise, o que pode ser uma limitação desta pesquisa. Essa subjetividade pode ser esclarecida com o exemplo daquelas pessoas que residem em condições desfavoráveis, mas que percebem esse local como excelente [46]. Apesar de o uso de questionários como estratégia de pesquisa quantitativa ser útil e ser usado na maioria das pesquisas de avaliação de satisfação de usuários [26,30], uma ressalva deve ser feita quanto a esse método, pois é considerado reducionista e não contempla a subjetividade da percepção da satisfa- 
ção [48]. Por ora, a abordagem aqui eleita aproxima-se do disposto na denominada teoria da atitude, onde a avaliação de determinado quesito é feita pelo indivíduo de modo positivo ou negativo [49]. Esperidião e Trad [24] criticam os modelos empregados nos estudos de avaliação de satisfação dos usuários e apontam para a necessidade de um consenso que promova validação e operacionalização desta medida. Esse objetivo foi contemplado na pesquisa de João et al. [32], onde os autores validaram as propriedades psicométricas de uma escala de satisfação dos pacientes relacionada especificamente com cuidados do médico de família, tendo-se chegado à conclusão positiva de sua validade e confiabilidade enquanto instrumento de avaliação para este fim.

Outra limitação deste estudo deve-se à realização das entrevistas na própria UBS. Mesmo que em sala reservada, os usuários podem não ter relatado tudo o que julgavam pertinente. Nesse sentido, Silva Filho et al. [46] afirmam que a abordagem dos usuários fora do espaço assistencial faz com críticas surjam mais facilmente. $\mathrm{O}$ desenho de estudo transversal também apresenta limitações em termos da dificuldade de estabelecimento da relação causal [47], mas justificase porque os autores não desejaram estabelecer um sentido de retrospecção ou de acompanhamento longitudinal dos pesquisados.
Em síntese, o grau de satisfação dos usuários da UBS Ferraria foi influenciado por fatores como raça/cor da pele, renda e escolaridade. Os usuários não brancos expressaram um grau de satisfação maior do que os usuários brancos. Os usuários com baixa escolaridade sabiam menos onde reclamar caso o atendimento não fosse satisfatório. Apesar da avaliação em geral ter sido positiva, alguns quesitos, como tempo de espera para o atendimento, foram identificados como não satisfatórios, merecendo medidas corretivas.

\section{NOTAS}

\section{Apoio financeiro}

Este estudo recebeu apoio financeiro da Fundação Araucária/ SETI/CPUP por meio de concessão de bolsa de Iniciação Científica.

\section{Declaração de conflito de interesses}

Os autores declaram não haver conflitos de interesses relevantes ao conteúdo deste estudo.

Contribuições dos autores

Todos os autores fizeram contribuições substanciais para concepção, ou delineamento, ou aquisição, ou análise ou interpretação de dados; e redação do trabalho ou revisão crítica; e aprovação final da versão para publicação.

Disponibilidade dos dados e responsabilidade pelos resultados

Todos os autores declaram ter tido total acesso aos dados obtidos e assumem completa responsabilidade pela integridade destes resultados.

\section{REFERÊNCIAS}

1. Brasil. Ministério da Saúde. Manual de planejamento no SUS. Brasília: Ministério da Saúde; 2016.

2. Brasil. Ministério da Saúde. Secretaria Executiva. Departamento de apoio a descentralização. O SUS no seu município: garantindo saúde para todos. Brasília: Ministério da Saúde; 2009.

3. Brasil. Ministério da Saúde. Gestão Municipal de Saúde: textos básicos. Rio de Janeiro: Ministério da Saúde; 2001.

4. Brasil. Ministério da Saúde. Secretaria de Assistência à Saúde. Departamento de Descentralização da Gestão da Assistência. Regionalização da Assistência à Saúde: aprofundando a descentralização com equidade no acesso: Norma Operacional da Assistência à Saúde: NOAS-SUS 01/02 e Portaria MS/GM no 373, de 27 de fevereiro de 2002 e regulamentação complementar/Ministério da Saúde, Secretaria de Assistência à Saúde. Departamento de Descentralização da Gestão da Assistência. - 2. ed. revista e atualizada. Brasília: Ministério da Saúde; 2002.

5. Brasil. Conselho Nacional de Secretários de Saúde. Para entender a gestão do SUS/Conselho Nacional de Secretários de Saúde. Brasília: CONASS; 2003.

6. Starfield B. Atenção primária: equilíbrio entre necessidades de saúde, serviços e tecnologia. Brasília: UNESCO/Ministério da Saúde; 2002.

7. Brasil. Ministério da Saúde. Secretaria de Assistência à Saúde. Coordenação de Saúde da Comunidade. Saúde da Família: uma estratégia para a reorientação do modelo assistencial. Brasília: Ministério da Saúde; 1997.

8. Arantes LJ, Shimizu HE, Merchán-Hamann E. Contribuições e desafios da Estratégia Saúde da Família na Atenção Primária à Saúde no Brasil: revisão da literatura. Ciênc saúde coletiva. 2016;21(5):1499-510. https://doi.org/10.1590/141381232015215.19602015

9. Crevelim MA, Peduzzi M. Participação da comunidade na equipe de saúde da família: é possível estabelecer um projeto comum entre trabalhadores e usuários? Ciênc saúde coletiva. 2005;10(2):323-31. https://doi.org/10.1590/S141381232005000200010

10. Moimaz SAS, Marques JAM, Saliba O, Garbim CAS, Zina LG, Saliba NA. Satisfação e percepção do usuário do SUS sobre o serviço público de saúde. Physis. 2010;20(4):1419-40. https://doi.org/10.1590/S0103-73312010000400019 
11. Trad LAB, Bastos ACS. O impacto sociocultural do Programa de Saúde da Família (PSF): uma proposta de avaliação. Cad Saúde Pública. 1998;14(2):429-35. https://doi.org/10.1590/S0102-311X1998000200020

12. Cotta RMM, Marques ES, Maia TM, Azeredo CM, Schott M, Franceschini SCC, Priore SEA. A satisfação dos usuários do Programa de Saúde da Família: avaliando o cuidado em saúde. Sci Med.2005;15(4):227-34.

13. Teixeira SA. Avaliação dos usuários sobre o programa de saúde da família em Vitória da Conquista - Bahia - Brasil. In: Fernandes AS, Seclen-Palacin JA, organizadores. Experiências e desafios da atenção básica e saúde familiar: caso Brasil. Brasília: Organização Pan-Americana da Saúde; 2004. p. 77-101.

14. Dos Santos Cruz J, De Almeida PF, Figueredo AH, Dos Santos AM. Avaliação do acesso à Estratégia Saúde da Família na perspectiva dos usuários no município de Santo Antônio de Jesus-Bahia, Brasil. Rev Salud Pública. 2017;19(5):641-8. https://doi.org/10.15446/rsap.v19n5.49356

15. Jorge MSB, Guimarães JMX, Vieira LB, Paiva FDS, Rocha de Silva D, Pinto AGA. Avaliação da qualidade do Programa de Saúde da Família no Ceará: a satisfação dos usuários. Rev baiana saúde pública. 2007;31(2):256-66.

16. Sena SLC, Ferreira LN, Oliveira RS, Kozmhinsky VMR. Acolhimento e satisfação do usuário na Estratégia de Saúde da Família: uma experiência de êxito. Rev APS. 2015;18(2):134-40.

17. Arruda CAM, Bosi MLM. Satisfação de usuários da atenção primária à saúde: um estudo qualitativo no Nordeste do Brasil. Interface (Botucatu). 2017;21(61):321-32. https://doi.org/10.1590/1807-57622015.0479

18. Oliveira MM, Pinto IC, Coimbra VCC, Oliveira EM, Alves PF, Cruz VD. Avaliação da aceitabilidade do usuário/família sobre a Estratégia Saúde da Família. Rev APS. 2017;20(2):159-66.

19. Protasio APL, Gomes LB, Machado LDS, Valença AMG. User satisfaction with primary health care by region in Brazil: 1st cycle of external evaluation from PMAQ-AB. Cienc Saude Colet. 2017;22(6):1829-44. https://doi.org/10.1590/141381232017226.26472015

20. Silva AS, Fracolli LA. Avaliação da Estratégia Saúde da Família: perspectiva dos usuários em Minas Gerais, Brasil. Saúde debate. 2014;38(103):692-703.

21. Araújo RL, Mendonça AVM, Sousa MF. Percepção dos usuários e profissionais de saúde no Distrito Federal: os atributos da atenção primária. Saúde debate. 2015;39(105):387-99. https://doi.org/10.1590/0103-110420151050002007

22. Senna MCM. Equidade e política de saúde: algumas reflexões sobre o Programa Saúde da Família. Cad Saúde Pública. 2002;18(Supl.):201-11. https://doi.org/10.1590/S0102-311X2002000700020

23. Silva SA, Baitelo TC, Fracolli LA. Avaliação da Atenção Primária à Saúde: a visão de usuários e profissionais sobre a Estratégia de Saúde da Família. Rev Latino-Am Enfermagem. 2015;23(5):979-87. https://doi.org/10.1590/01041169.0489 .2639

24. Esperidião MA, Trad LAB. Avaliação de satisfação de usuários: considerações teórico-conceituais. Cad Saúde Pública. 2006;22(6):1267-76. https://doi.org/10.1590/S0102-311X2006000600016

25. Weiss GL. Patient satisfaction with primary medical care. Evaluation of sociodemographic and predispositional factors. Med Care. 1988;26(4):383-92. https://doi.org/10.1097/00005650-198804000-00007

26. Esperidião M, Trad LAB. Avaliação de satisfação de usuários. Ciênc saúde coletiva. 2005;10(Supl.):303-12.

27. Medeiros FA, de Araújo-Souza GC, Albuquerque-Barbosa AA, Clara-Costa Ido C. Basic health unit embracement: focusing on user satisfaction. Rev Salud Publica (Bogota). 2010;12(3):402-13. https://doi.org/10.1590/S0124-00642010000300006

28. Almeida RS, Nogueira LA, Bourliataux-Lajoine S. Analysis of the user satisfaction level in a public physical therapy service. Braz J Phys Ther. 2013;17(4):328-35. https://doi.org/10.1590/S1413-35552013005000097

29. Murillo C, Saurina C. Measurement of the importance of user satisfaction dimensions in healthcare provision. Gac Sanit. 2013;27(4):304-9. https://doi.org/10.1016/j.gaceta.2012.12.015

30. Wu J, Zhang S, Chen H, Lin Y, Dong X, Yin X, Lu Z, Cao S. Patient satisfaction with Community Health Service Centers as gatekeepers and the influencing factors: a cross-sectional study in Shenzhen, China. PLoS One. 2016;11(8):e0161683. https://doi.org/10.1371/journal.pone.0161683

31. Hone T, Gurol-Urganci I, Millett C, Başara B, Akdağ R, Atun R. Effect of primary health care reforms in Turkey on health service utilization and user satisfaction. Health Policy Plan. 2017;32(1):57-67.

32. João AR, Monteiro MJFSP, Pereira MCARS, Rodrigues VMCP. Versão portuguesa da escala de satisfação dos pacientes com os cuidados do médico de família - SatMF17: validação psicométrica. Sci Med. 2018;28(3):ID31286. https://doi. org/10.15448/1980-6108.2018.3.31286

33. Brasil. Ministério do Planejamento, Orçamento e Gestão. Instituto Brasileiro de Geografia e Estatística - IBGE. Cidades@ [Internet]. Brasília; 2017 [cited 2017 Apr 24] Available from: http://cidades.ibge.gov.br/xtras/perfil. php?lang=\& codmun=410420

34. Prefeitura Municipal de Campo Largo. Conselho Municipal de Desenvolvimento Urbano e Meio Ambiente. Secretaria Municipal de Desenvolvimento Urbano. Plano Diretor do Município de Campo Largo 2016-2026. Processo de Revisão 2015 [internet]. Curitiba; 2015 [cited 2017 Apr 24] Available from: http://planodiretor.campolargo.pr.gov.br/

35. Brasil. Ministério da Saúde. Secretaria-Executiva. Departamento de Regulação, Avaliação e Controle de Sistemas. PNASS: Programa Nacional de Avaliação de Serviços de Saúde/Ministério da Saúde, Secretaria-Executiva, Departamento de Regulação, Avaliação e Controle de Sistemas. Brasília: Ministério da Saúde; 2015. 
36. Campos RTO, Ferrer AL, Gama CAP, Campos GWS, Trapé TL, Dantas DV. Avaliação da qualidade do acesso na atenção primária de uma grande cidade brasileira na perspectiva dos usuários. Saúde debate. 2014;38:252-64. https:// doi.org/10.5935/0103-1104.2014S019

37. Santos MP. Avaliação da qualidade dos serviços públicos de atenção a saúde da criança sob a ótica do usuário. Rev bras enferm. 1995;48(2):109-19. https://doi.org/10.1590/S0034-71671995000200002

38. Santiago RF, Mendes ACG, Miranda GMD, Duarte PO, Furtado BMASM, Souza WV. Qualidade do atendimento nas Unidades de Saúde da Família no município de Recife: a percepção doa usuários. Ciênc saúde coletiva. 2013;18(1): 35-44. https://doi.org/10.1590/S1413-81232013000100005

39. Bicalho CS, Lacerda MR, Catafesta F. Refletindo sobre quem é o cuidador familiar. Cogitare Enferm. 2008;13(1): 118-23. https://doi.org/10.5380/ce.v13i1.11972

40. Queiroz MS. Estratégias de consumo em saúde entre famílias trabalhadoras. Cad Saúde Pública. 1993;9(3):279-82. https://doi.org/10.1590/S0102-311X1993000300015

41. Barbosa YO, Menezes LPL Santos JMJ, Cunha JO, Menezes AF, Araújo DC, Albuquerque TIP, Santos AD. Acesso dos homens aos serviços de atenção primária à saúde. Rev enferm UFPE on line. 2018;12(11):2897-905. https://doi. org/10.5205/1981-8963-v12i11a237446p2897-2905-2018

42. Pinheiro RS, Viacava F, Travassos C, Brito AS. Gender, morbidity, access and utilization of health services in Brazil. Ciênc saúde coletiva. 2002; 7(4): 687-707. https://doi.org/10.1590/S1413-81232002000400007

43. Coyle J. Exploring the meaning of "dissatisfaction" with health care: the importance of "personal identity threat". Sociol Health Illn. 1999;21(1):95-123. https://doi.org/10.1111/1467-9566.00144

44. Boccolini CS, Boccolini PMM, Damacena GN, Ferreira APS, Swarcwald CL. Fatores associados à discriminação percebida nos serviços de saúde do Brasil: resultados da Pesquisa Nacional de Saúde, 2013. Ciênc saúde coletiva. 2016;21(2):371-8. https://doi.org/10.1590/1413-81232015212.19412015

45. Viegas APB, Carmos RF, Luz ZMP. Fatores que influenciam o acesso aos serviços de saúde na visão de profissionais e usuários de uma unidade básica de referência. Saude soc. 2015;24(1):100-12. https://doi.org/10.1590/S010412902015000100008

46. Silva Filho JF, Cavalcanti MT, Cadilhe GM, Vilaça AT, Avancini E, Machado HO, Perecmanis L, Maisonnette MJ, Rocha EC, Carreteiro RO, Mozinho GMP, Pitta AM. Avaliação de qualidade de serviços de saúde mental no Município de Niterói - RJ. A satisfação do usuário como critério avaliador. J Bras Psiquiatr. 1996;45(7):393-402.

47. Belbasis L, Bellou V. Introduction to epidemiological studies. Methods Mol Biol. 2018;1793:1-6. https://doi. org/10.1007/978-1-4939-7868-7_1

48. Trad LAB, Bastos ACS, Santana EM, Nunes M. O estudo etnográfico da satisfação de usuário do Programa de Saúde da Família (PSF) na Bahia. Ciênc saúde coletiva. 2002;7(3):581-9. https://doi.org/10.1590/S1413-81232002000300015

49. Linder-Pelz S. Toward a theory of patient satisfaction. Soc Sci Med. 1982;16(5):577-82. https://doi.org/10.1016/02779536(82)90311-2 $\mathrm{C}$ 